\title{
Bayesian Model-based Personalized Recommendation Service
}

\author{
Jeong-Sig Kim ${ }^{1}$, Eung-Sung $\mathrm{Kim}^{2}$ and Jin-Hong Kim ${ }^{3}$ \\ ${ }^{1,2}$ Department of Computer \& Mobile Convergence, \\ Gyeonggi College of Science and Technology \\ ${ }^{3}$ College of Information and Communication Engineering, \\ Sungkyunkwan University
$\left\{{ }^{1}\right.$ arius70, ${ }^{2}$ imagecap $\} @ g t e c . a c . k r,{ }^{3}$ jinhkm@skku.edu
}

\begin{abstract}
Personalized recommendation service as recommendation systems provides software tools and techniques supporting suggestions to a user. The suggestions provided are aimed at supporting their users in various decision making processes such as what items to buy, what music to listen, what profiles to browse, or what news to read. Eventually, personalization technology has the potential to optimize service for each user's needs and behaviors. This service is to allow user to customize the service itself, and proactively tailor services based on information from past service historical data. After all, these approaches function best when user knows what they want and require, and when their behaviors and preferences remain consistent over context. In this paper, we present personalized recommendation service as a Bayesian Modeling on commerce market.
\end{abstract}

Keywords: Recommendation System, Bayesian Modeling, Personalization Technology

\section{Introduction}

Services are increasingly becoming technology-based, delivered by computing technology commerce platforms. Technology-based allow for efficient delivery of services that were previously provided only by user [1]. With the development of intelligent ubiquitous computing, artificial intelligent, and big data environment have emerged as well with influence their experience. Personalization has taken on new importance in these technology-based services, as thy offer unpredicted opportunities to optimize services for individuals [2]. Unlike user service providers, who have limited attention, time, and resources, computing technology can easily keep records of user intention and personalize its services for each individual with affordable costs. Useroriented personalization suggests many reasons why personalized services would be more effective than mass-produced services. Accordingly, these service domains can be characterized as preference-based [3]. That is, user's choices about and uses of services are mainly guided by what they like and are interested in. As these are part of their everyday choices, users tend to have well-defined preferences for these services. Recently, however, technology-based services are moving beyond these preferencesbased domains. For example, in health and education, the efficacy of services is not just determined by whether people like the service or not, but also by whether service solutions satisfy user needs [4]. This knowledge is often derived from domain expertise. In addition, users may not have formed preferences on these services, as they lack domain knowledge and do not deal with these choices every day. Accordingly, in our research paper, we provide new design principles, knowledge about user behaviors, and research methods for designing personalized technology-based services. 


\section{Approach to Personalization Service}

\subsection{Definition of Personalization}

Over the past two decades, many definitions of personalization have been proposed in the field of business, marketing and more. The common thread in these definitions is that personalization involves activities of tailoring technology service offerings and interaction to achieve certain outcomes [5]. In addition, these provide the most comprehensive framework, which describes different design dimensions of personalization for technology-based services. These dimensions include: 1) Personalization is to systemdriven and user-driven service that is customizing some feature of a product or service so that the customer enjoys more convenience, lower cost or some other benefit. 2) Personalization in general means matching one object's nature with one subject's needs. 3) Personalization is a process that changes the functionality, interface, information content, or distinctiveness of a system to increase its personal relevance to the individual. 4) Personalization refers to the tailoring of products and purchase experience to the tastes of individual consumers based upon their personal and preference information. Therefore, personalization is critically dependent on vendors' ability to acquire and process consumer information, and on consumers' willingness to share information and use personalization services [6]. In these cases, in personalization, a web site provides an array of choices for the users to modify a web site's look and feel (i.e. is a user-driven process). Relevant content based on the preferences of groups of users is provided in adaptation (i.e. according to the country of web users) [7]. Personalization is a process of providing relevant content based on individual user preferences, and personalized web sites obtain preference information implicitly by tracking customer purchases or usage habits. There are three types of personalization: user-driven personalization when the user specifies in advance the desired web layout and content that matches her interests and preferences with the tools and options provided. In transaction-driven personalization, an online merchant generates the personalized layout and content, and thus personalization is driven by previous transactions [8]. Context-driven personalization employs an adaptive mechanism to personalize content and layout for each individual user based on the context and inference of users' processing objectives in real time.

\subsection{Personalization Technology}

Technology-based services can be customized by users or automatically personalized by systems. With the emergence of intelligent technology that utilizes user's clickstream data online, digital records such as electronic health records, and sensor data, systemdriven personalization driven by systems is becoming more and more prevalent, and new research issues regarding control and user autonomy are emerging [9]. 1) User-Driven Personalization. User-driven personalization, often called customization, has received much attention in research, especially after the introduction of interactive technology. Interactive technologies such as websites or software allow users to customize numerous features to best fit their needs and preferences with relatively little cost. However, many studies downplay the drawbacks and boundary cases of user-driven personalization. Some have shown that customization can be too labor-intensive and complex for users and those users do not always have good insights into their needs. 2) System-Driven Personalization. System-driven personalization is personalization in which systems implicitly and often automatically tailor services based on users' observed behaviors or inputs obtained through surveys. System-driven personalization has been popular since the mid-2000s, and its popularity has increased sharply [10]. This increase is in part due to the development of multiple computational techniques on recommender systems, such as content-based filtering, and user-modeling. These techniques use data from massive social networks or users' clickstream data to infer individuals' characteristics and personalize 
websites or interactive technology services, often without users' explicit efforts or control. For example, Facebook, Amazon, and Google present each individual with personalized lists of recommended friends, books, and search results, respectively. System-driven personalization is thought to reduce information overload, and is presumed to increase the profits of many online content-providing companies [11]. More recently, the exponential increase in digital data sets on human behaviors and choices has allowed for "big data analytics", expanding domains where system-driven personalization can be applied, such as entertainment, health, and education [12]. However, to our knowledge, there is little research investigating how systems can adapt their personalization techniques over time to support people's changing experiences and contexts.

\subsection{Personalization Service}

Service has been of interest to the business and marketing community for nearly three decades. Increasingly more attention, both in academia and in practice, has been given to service in the 2000s, with emphases on the "experience economy" and the digital technologies that transform service delivery [13]. So to speak, a service is an activity or series of activities of more or less intangible nature that normally, but not necessarily, take place in interactions between the customer and service employees and/or physical resources or goods and/or systems of the service provider, which are provided as solutions to customer [14].

Service, as a view point, renders all economic activities as value creation rather than product exchange, which makes service the dominant form of economic activities. This perspective encourages designers to primarily focus on creating value for both customers and providers throughout use experience over time, rather than focusing on creating new product features or details. Exchange of products has been considered the basis of the economy; thus many researchers in business and marketing have sought to define the characteristics of service by comparing them with characteristics of products [15]. The most commonly discussed properties of services include the following; 1) Intangibility. While tangible goods may be included in the delivery of services, the essence of a service is the intangibility of the phenomenon itself. 2) Activities or a series of activities. A service is a not a thing but a series of activities or processes - which, moreover, are produced and consumed simultaneously. 3) Co-creation/production. The customer is not only a receiver of the service; the customer participates as a production resource as well. 4) Multi-stakeholders and multiple platforms. A service is often delivered through multistakeholders, including providers and customers, via multiple platforms.

\section{Recommendation Systems}

Recommendation Systems (RS) are used in wide variety of contexts. These contexts range from online shops to personalized contents. RS can be found in classical web-based appliances and lately also in mobile devices [16-18]. Generally, we could define that a RS can be used in every context where a user is to choose among several different options. The system assists the user to select one good-fitting item. Content-based recommendation systems provide items "by comparing representations of content contained in an item to representations of content that interests the user." In most cases, a keyword profile is built. Apparently, this works well in text domains but not in domains where there is not much content associated with the items or where a computer cannot easily analyze this content [19]. Collaborative filtering systems compute profile similarity between the other users and the target user "by comparing users" opinions of items." Profile similarity is usually computed by comparing rating-vectors with various distance metrics with user correlation or cosine similarity [20]. 


\subsection{Content-Based Filtering System}

Content-based filtering systems utilize machine learning techniques such as naïve bayes to analyze WWW pages, Usenet News, Email, and other types of electronic content amenable to automatic textual analysis. Also, in content-based filtering systems, the user profile and the document profile are represented as a weighted vector in terms of keywords. In recommendation systems, in which content-based filtering is applied, recommendations are made for a user based solely on a profile built from analyzing the content of items that the user has rated in the past. InfoFinder and WebWatcher are examples of such systems [21]. Content-based filtering systems have several defects. The first problem is that only a very shallow analysis of specific content can be applied. As well as characteristics of the content itself, there are many other aspects of the content, such as public quality and time consumed in loading content. The system ignores such factors. The second problem is over-specialization. When the system can only recommend items scoring highly against a user's profile, the user is restricted to seeing items similar to those already rated.

\subsection{Collaborative Filtering System}

Collaborative filtering systems select items for a particular user when they are also relevant to other similar users. In addition, collaborative filtering systems provide predicted information applicable with user preferences through a correlation engine based on clarified information and estimating the degree of user preference. A recommendation system, utilizes a collaborative filtering system, but does not analyze an item at all. The system recommends items for a user solely based on similarities to other users. GroupLens and Ringo are examples of such systems [22]. A collaborative recommendation system solves problems relating to content-based recommendations. Using other user's ratings allows us to deal with any kind or content and receive items with dissimilar content to those seen in the past. Since other user's feedback influences recommendations, there is the potential to maintain effective performance even when given fewer ratings from any individual user. Nevertheless, this system has some problems. The first problem is that the coverage of ratings could be too sparse, resulting in insufficient recommendations [23]. Recommendations may not be accurate in some cases, for example, in the case of new items being inserted into the database, or that the number of users is too small relative to the number of the items in the system. The second problem is that there will not be many like-minded users for a user whose tastes are unusual, compared with the rest of the users.

\subsection{Recommendation Process}

Information recollection will be the base for the entire recommendation system which the recollection of users' personal preferences and information about items such as metadata, features extracted directly [24]. This process is not performed by the recommendation system itself because the information collected presents incongruence or contradiction then the system will not be able to produce regular recommendations. Hence, proper attention should be adopted in collecting information that truly reflects the preferences of the users, or information that truly represent the items as following bellows in Figure1. 1) Selection. consists of determining "which items are interesting or relevant enough for a user and removes all other items from the retrieved set of items". The way the selection of the items is done depends strictly on the approach taken to find items that are similar to the ones the user consider relevant. Therefore, the key concept is defining similarity between items [25]. The concept of similarity could be defined in terms of other users' profiles, of features extracted from the items, or in terms of metadata associated with the items. 2) Transformation. The main objective of the transformation is to perform some modifications to the items retrieved and proposed to addresses transformations such 
as summarization, change in the quality of the items, creation of thumbnails or snapshots for its further presentation. 3) Structure. The structure that the user will use to navigate through the different recommended items is related with the construction and organization. In addition to the above grouping the items according to certain characteristics, sorting the groups of items, sorting items inside these groups, linking items that have some relationship, etc. can be included. 4) Recommendation Process. The recommendation process is associated with the presentation of the different retrieved and structured items to the final user. It deals with issues such as layouts, document formats, colors, fonts, and presentation medium. This process should be designed and executed carefully since the user will interact with the system by means of its results. 5) Feedback. The kind of feedback obtained from the user could be either implicit or explicit. In explicit feedback the user provides the system with information about how relevant the recommended items are [26]. On the other hand implicit feedback is obtained from the user by analyzing his usage behavior. For instance, how much time he spends in looking at the retrieved items.

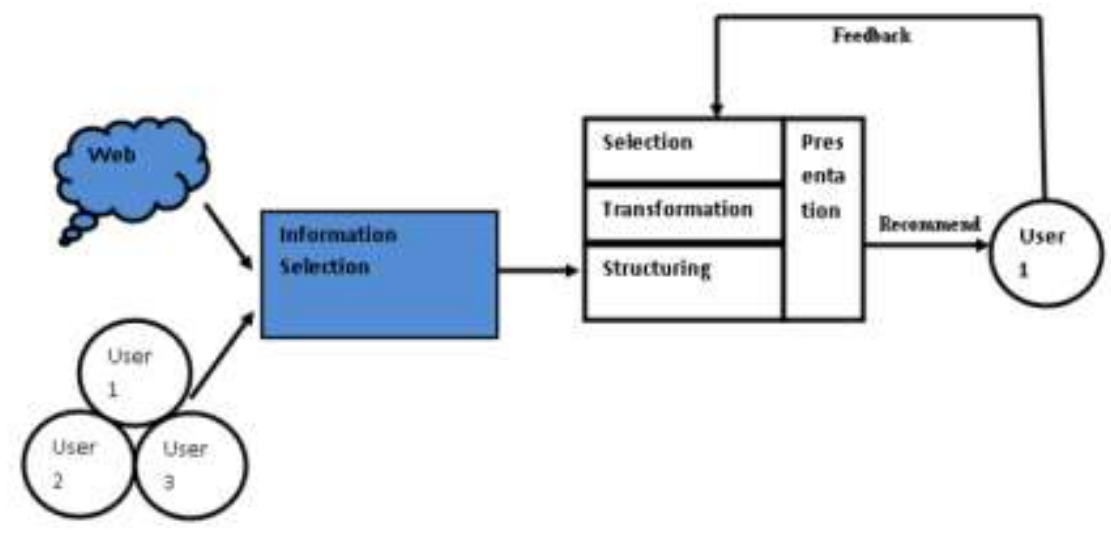

Figure 1. Recommendation Process

\section{Bayesian Reasoning}

In this paper, we explain topics related to what we refer to as Bayesian reasoning, which is reasoning in domains with high levels of uncertainty and where partial beliefs about probabilities are updated as more evidence is observed. We first present Bayes' theorem, also referred to as Bayes' rule, which lies at the core of Bayesian reasoning, before we move on to Bayesian networks which offer advantages when it comes to modelling in uncertain domains [27]. In the last part of this section we look at different recommender systems presented in the literature which use Bayesian reasoning to generate recommendations for users.

\subsection{Bayes' Theorem}

Bayesian theorem explains the relation between a conditional probability and its inverse conditional probability. If $\mathrm{H}$ is a hypothesis and $\mathrm{E}$ is the observed evidence for or against $\mathrm{H}$, the theorem states that: $P(H \mid E)=P(E \mid H) P(H) / P(E)$. Bayes' theorem is helpful in probabilistic reasoning as it provides us with a method to update our beliefs about a hypothesis when new information or evidence becomes available. 


\subsection{Bayesian Networks}

Bayesian networks are graphical models commonly used in probabilistic reasoning and artificial intelligence. They were first model dependency relations between variables in uncertain domains. A Bayesian network is defined by the following way: 1) it consists of a set of variables, represented as nodes, and a set of directed edges between variables. 2) For each variable it is defined a finite set of mutually exclusive states. 3) The network contains no cycles, hence it is a directed acyclic graph. 4) Each variable $X$, with parents

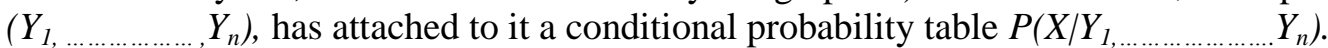

In a Bayesian network, we say that a node $X$ that has a directed link to another node $Y$, is a parent of the latter node $Y$. This intuitively means that $X$ has a direct influence on $Y$. In general, the edges of the network encode dependencies between variables. A key assertion, which is made clear through the encoding, is that variables are conditionally independent of its non-descendants given its parents [28]. One of the biggest advantages of Bayesian networks is their capability of representing joint probability distributions in a compact way, because of the chain rule for Bayesian networks. Let $X=\left(X_{1}, \ldots \ldots \ldots \ldots \ldots, X_{n}\right)$ be represented by a Bayesian network and let the function Parents $\left(X_{i}\right)$ denote the set of variables in the network which have a directed edge to variable $X_{i}$. Then, according to the chain rule for Bayesian networks, the Bayesian network specifies a unique joint probability distribution $P(X)$.

\subsection{Bayesian Reasoning in Recommendation Systems}

In model-based recommender systems, both collaborative and content-based, some form of Bayesian reasoning is often applied to generate recommendations. We here provide a short overview of how Bayesian reasoning has previously been used in recommender systems. One example of using Bayesian networks in a model-based recommender system approach based on collaborative filtering. They use rating data to learn a Bayesian network where each item is represented by a node, and directed arrows between items signify how liking of items influence liking of other items. The network is used to create probabilistic decision trees for each item, where leaf nodes are likelihoods of the target user liking the target item, and intermediary decisions are based on the target user's view on the parent items of the target item from the network.

\section{Predictive Bayesian Model for Recommendation Service}

We propose an application of the Bayesian Classifier (BC) to the collaborative filtering problem space. The $\mathrm{BC}$ is one of the most successful machine learning algorithms in many classification domains [29][30]. In spite of its simplicity, it's shown to be competitive with other complex approaches, especially in text categorization tasks.

Bayesian Preference Elicitation (BPE). Preference elicitation is an important component of RS that propose items or services from a potentially large set of available choices but due to practical constraints may only query a limited number of preferences. BPE task consists of user utility model. Utilities are modeled over a $D$-dimensional

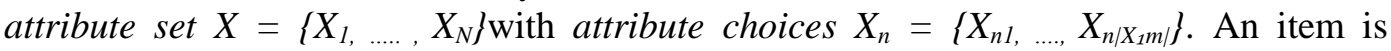
described by its attribute choice assignments $\mathrm{x}=\left(\mathrm{x}_{1, \ldots}, \mathrm{X}_{\mathrm{N}}\right)$ where $\mathrm{x}_{\mathrm{N}} \in \mathrm{X}_{\mathrm{n}}$. In our model, an attribute weight vector $\mathrm{w}=\left(\mathrm{w}_{11}, \ldots, \mathrm{w}_{\left.1\left|\mathrm{x}_{1}\right|, \ldots \ldots . . .\right)}\right)$ described by its attribute choice assignments $\mathrm{x}$ where $\mathrm{x}_{\mathrm{n}} \in \mathrm{X}_{\mathrm{n}}$.

Query User Response Model (QUM). We focus on pairwise comparison queries known to require low cognitive load for users, hence reducing noise in the elicitation process. We use $Q_{i j}=\{i \supset j, i \subset j, i \sim j\}$ to represent a pairwise comparison query indicating the user's preferences of item $x_{j}$. Depending on the user's attribute weight vector $\mathrm{w}$ and the corresponding item utilities, $u(i \mid w)$ and $u(j \mid w)$, the user's response $q_{i j} \in Q_{i j}$ indicates. 


\subsection{Conceptual PBM}

The systems that we propose in this paper approach of RS, so we have to consider two different information sources: the content-based knowledge and the collaborative component. With respect to the content component we typically have a large number $m$ of items $I=\left\{I_{1}, I_{2}, \ldots \ldots I_{m}\right\}$ and a large number $l$ of features $F=\left\{F_{l}, F_{2}, \ldots . F_{l}\right\}$, each item has been described by a set of attributes or features.

Content based Component. This component contains the set of $\mathrm{m}$ features, $F$, and the set of $l$ items, $I$. There exists a feature node $F_{k}$ for each feature used to describe a product and also, there exist a node for each product in the system.

Collaborative filtering Component. This component is used to relate a given user with other users with similar tastes. These relationships should be directly modeled in the PBM by means of the inclusion of arcs between any two related users. Thus, a similarity between the preferences of user $U_{1}$ and user $U_{2}$ has been found, an arc connecting both nodes will be included in the PBM as following with Figure 2.

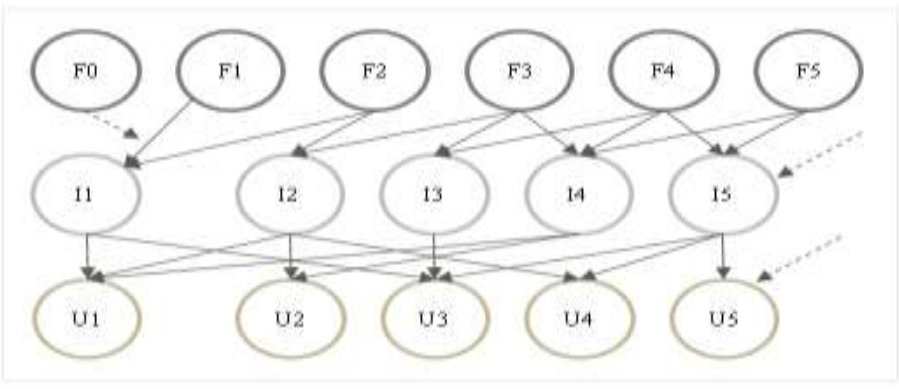

Figure 2. Recommendation System Topology for BPM

We are described the different preprocessing steps induced by a data analysis. We will only be kept once the algorithm is built, the terms 'customer' and 'household' will both state for the same unique entity: the user from the panel data. This will remain true until said otherwise. We used the general matrix notation, with customers as the rows and products as the columns. Implicitly, this notation surmised that the products offered by the retailers were the same. In practice this is not true. Actually there are more than ten thousand different products ID in the dataset. It is interesting to plot the revenue generated by the sales for each product, as shown on Figure 3.

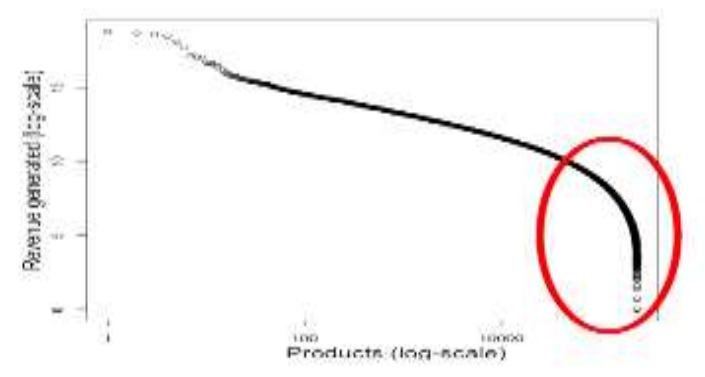

Figure 3. Products on a Log-log Scale

A first rational thing to do is to remove this very long tail, which denotes seasonal products, special offers or simply scarce products sold in a minority of retailers. Just before removing this heavy tail it is interesting to look at the retailers revenues which also display a long tail phenomenon, as following in Figure 4. The discrepancy in the retailers' revenues is also due to outliers which are online retailers, retailers which are not primarily focused on consumer products and so on. Another step of preprocessing is thus needed. 
Also, encouraged by the company which provided the data, we developed a special taxonomy of the products, namely the 'recommender ID', which is the fusion of the product category and the brand. The idea is that different product IDs state for the same product category.

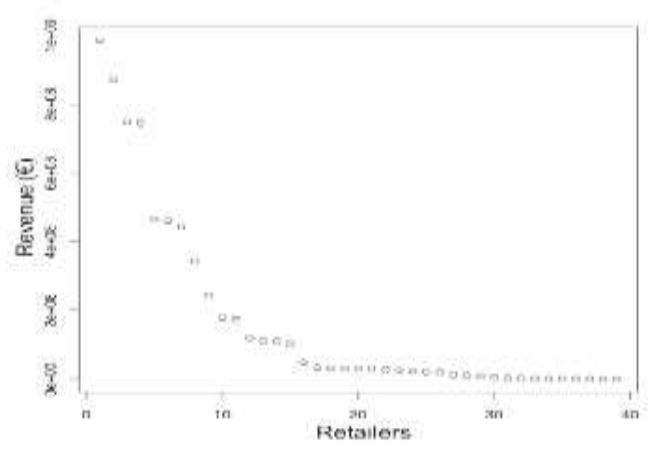

Figure 4. Revenue Generated on Retailer

Accordingly, we have sum up the Bayesian Modeling for Recommendation service gives a very good performance with few relevant product. On the contrary the algorithm explodes when the number of products increases, due to the fact that the numbers of 0 for the products in the tail overwhelm the number of 1 as following in Figure5.
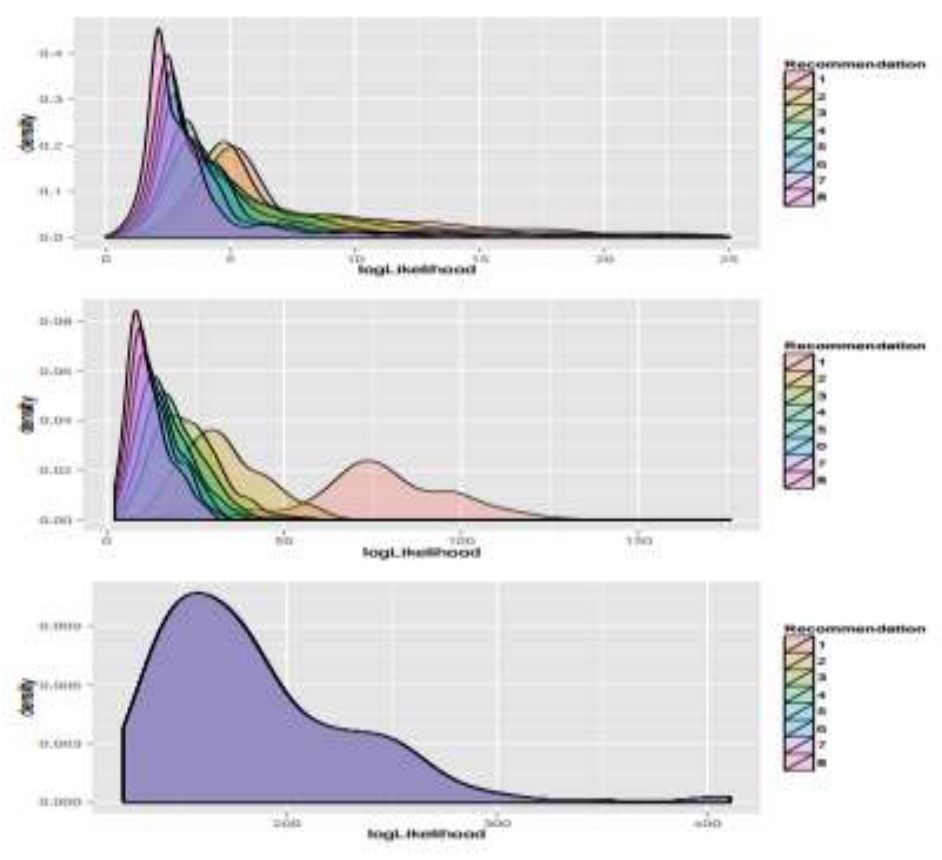

Figure 5. Bayesian Modeling for Recommendation Service Performance

\section{Conclusions}

A general BPM for RS has been proposed in this paper, additionally, learning for the Content-based component should be created. In order to be able to do this, we should afford the necessary data. This data should contain usage statistics, when the user with content descriptions for the assets should be available in personalized data. Furthermore, the relation of implicit and explicit feedback of users should be researched. Accordingly, 
a various content component and dynamic environment, we should be achieve by integrating new feedback methods, user profiling information, query's pattern with Big Data of real world. Also, a hybrid approach combining both collaborative and contentsbased should be consider with hybrid platforms.

\section{References}

[1] J. Kim and E. Lee, "XQuery Pattern for Semantic Web based Personalization Recommender Service", International Journal of Information Technology, vol. 12, no. 6, (2006), pp. 108-116.

[2] J. Kim and E. Lee, "A Study of XFCM Method for Personalization Recommender System: User Profile, Web Category and Product", GESTS International Transaction on Computer Science and Engineering, vol. 5, no. 1, (2005), pp.89-99.

[3] E. Lee and J. Jin, "A Next Generation Intelligent Mobile Commerce System”, LNCS 3026, SpringerVerlag, (2004), pp. 320-331.

[4] E. Lee and S. Jang, "A User Adaptive Intelligent Mobile Commerce System with a Middlet Application”, LNCS 2412, Springer-Verlag, (2002), pp.193-198.

[5] I. G. Jeon and E. S. Lee "I-Pro: Intelligent Product Finding System for Electronic Commerce", International Journal of Electronic Commerce, M.E. Sharpe, vol. 4, no. 2, (2000), pp. 83-98.

[6] J. Basilico and T. Hofmann, "Unifying collaborative and content-based filtering", In Proceedings of the $21^{\text {st }}$ International Conference on Machine Learning, Page 9, New York, NY, USA, ACM, (2004).

[7] D. M. Blei, A. Y. Ng, and M. I. Jordan, "Latent Dirichlet allocation", Journal of Machine Learning Research, (2003), pp. 993-1022.

[8] E. Bonilla, S. Guo, and S. Sanner, "Gaussian process preference elicitation", In J. La_erty, C. K. I. Williams, J. Shawe-Taylor, R. Zemel, and A. Culotta, editors, Advances in Neural Information Processing Systems, vol. 23, (2011), pp. 262-270.

[9] C. Boutilier, R. Das, J. Kephart, G. Tesauro, and W. Walsh, "Cooperative negotiation in autonomic systems using incremental utility elicitation", In Proceedings of the 19th Conference Annual Conference on Uncertainty in Artificial Intelligence, San Francisco, CA, Morgan Kaufmann, (2003), pp. 89-97.

[10] C. Boutilier, R. Patrascu, P. Poupart, and D. Schuurmans, "Regret-based utility elicitation in constraintbased decision problems", In Proceedings of the $19^{\text {th }}$ international joint conference on Artificial intelligence, San Francisco, CA, USA, Morgan Kaufmann Publishers Inc., (2005), pp. 929-934.

[11] C. Boutilier, R. S. Zemel, and B. Marlin, "Active collaborative filtering", In Proceedings of the Nineteenth Annual Conference on Uncertainty in Artificial Intelligence, Acapulco, Mexico, Morgan Kaufmann, (2003), pp. 98-106.

[12] J. Canny, "Collaborative filtering with privacy via factor analysis", In Proceedings of the 25th Annual International ACM SIGIR Conference on Research and Development in Information Retrieval, ACM, (2002), pp. 238-245.

[13] M. A. Casey, R. Veltkamp, M. Goto, M. Leman, C. Rhodes, and M. Slaney, "Content-based music information retrieval: Current directions and future challenges", Proceedings of the IEEE, vol. 96, no. 4, (2008), pp. 668-696.

[14] W. Sen, Z. Xiaonan, and D. Yannan, "A Collaborative Filtering Recommender System Integrated with Interest Drift basd on Forgetting Function", International Journal of u- and e- Service, Science and Technology, SERSC, vol. 8, no. 4, (2015), pp. 247-264.

[15] X. Yang, and P. Liu, "Collaborative Filtering Recommendation using Matrix Factorization A MapReduce Implementation", International Journal of Grid and Distributed Computing, SERSC, vol. 7, no. 2, (2014), pp. 1-10.

[16] J. E. Garcia and A. C. R. Paiva, "REQ Analytics: A Recommender System for Requirements Maintenance", International Journal of Software Engineering and Its Applications, SERSC, vol. 10, no. 1, (2016), pp. 129-140.

[17] S. Verma and A. K. Manjhvar, "Optimized Ranking Based Recommender System for Various Application Based Fields", International Journal of Database and Theory and Application, SERSC, vol. 9, no. 2, (2016), pp. 137-144.

[18] C. Yang, J. Wang, M. Yuan and C. Lei, "A Novel Intelligent Recommendation Algorithm based on Web Data Mining Technique under the Background of Deep Neural Network", International Journal of Security and Its Applications, SERSC, vol. 10, no. 2, (2016), pp. 437-450.

[19] X. G. Wang, C. H. Li and T. Sun, "Domain Knowledge Actively Recommendation System Based by Process-Driven and Rough Set", International Journal of Database Theory and Application, vol. 9, no. 5 (2016), pp. 213-220.

[20] H. Lee and J. Kwon, "Personalized TV Contents Recommender System Using Collaborative Context tagging-based User's Preference Prediction Technique", International Journal of Multimedia and Ubiquitous Engineering, SERSC, vol. 9, no. 5, (2014), pp. 231-240.

[21] H. Lee and J. Kwon, "Efficient Recommender System based on Graph Data for Multimedia Application", International Journal of Multimedia and Ubiquitous Engineering, SERSC, vol. 8, no. 4, (2013). 
[22] F. Li, J. Sun and X. Zhang, "Study on the Key Technology of Personalized Recommendation of Casebased Reasoning", International Journal of u- and e- Service, Science and Technology, SERSC, vol. 8, no. 4 (2015), pp. 377-382.

[23] H. Lee and J. Kwon, "Social Clustering-based Similar User Indexing for Large Recommender System", International Journal of Multimedia and Ubiquitous Engineering, SERSC, vol. 9, no. 9, (2014), pp. 357370.

[24] A. Mathur, S. G. Shanmugam and N. Ch. S. N. Iyengar, "Agent Based Yoga Recommendation System for Better Health”, International Journal of Bio-Science and Bio-Technology, SERSC, vol. 8, no. 3, (2016), pp. 239-248.

[25] C. He, Y. Tang, Z. Yang, K. Zheng and C. Chen, "SRSH: A Social Recommender System based on Hadoop", International Journal of Multimedia and Ubiquitous Engineering, SERSC, vol. 9, no. 6, (2014), pp. 141-152.

[26] J. Kim and S. T. Hwang, "Big Data Platform for a System Recommendation in Cloud Environment", International Journal of Software Engineering and Its Applications, SERSC, vol. 9, no. 12, (2015), pp. 133-142.

[27] J. Kim and S. T. Hwang, "Adaptive Consistency Approaches for Cloud Computing Platform", International Journal of Software Engineering and Its Applications, SERSC, vol. 9, no. 7, (2015), pp. 127-134.

[28] J. S. Kim, E. S. Kim and J. Kim, "Towards Conceptual Predictive Modeling for Big Data Framework", International Journal of Software Engineering and Its Applications, vol. 10, no. 1, (2016), SERSC, pp. $35-42$.

[29] J. Kim and E. Lee, "XFCM-XML Based on Fuzzy Clustering and Merging- Method for Personalized User Profile Based on Recommendation System of Category and Product", Lecture Note in Computer Science, AWCC, Springer-Varlag, (2004), pp.323-330.

[30] J. Kim and E. Lee, "Semantic Web Recommender System Based Personalization Service for User XQuery Pattern”, Lecture Note in Computer Science, WINE, Springer-Verlage, (2005), pp. 848-857.

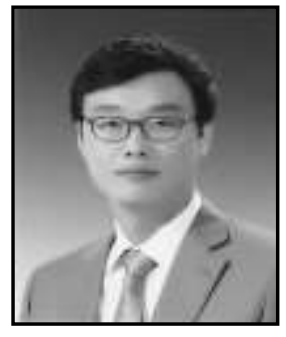

Jeong-Sig Kim, received the $\mathrm{Ph}$. D. degree in Electrical Electronic and Computer Engineering from SungKyun-Kwan University, Rep. of Korea in 2007. He worked the development of computing system in enterprise knowledge strategy consulting during $2005 \sim 2007$ and the DRM(Digital Rights Management) development in DRM-inside during 2007 2012. Since 2012, He is currently an assistant professor in the Department of Computer and Mobile Convergence at Gyeonggi College of Science and Technology, Rep. of Korea. His research interests are Error Resilient Coding, Digital Signal Processing, DRM System, Multimedia Processing \& Communications System, Big Data System and Software Engineering.

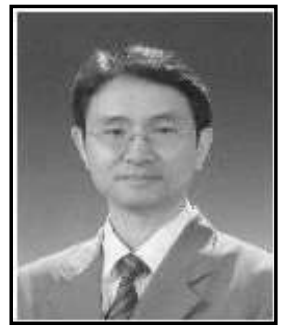

Eung-Sung Kim, received the $\mathrm{Ph}$. D. degree in Electronic Engineering from SungKyun-Kwan University, Rep. of Korea in 1998. Since 2000, He is currently a professor in the Department of Computer and Mobile Convergence at Gyeonggi College of Science and Technology, Rep. of Korea. His research interests are Image and Video Processing, Digital Signal Processing, Multimedia Communications, and Neuron Network.

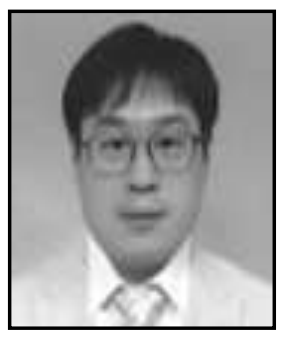

Jin-Hong Kim, received the $\mathrm{Ph}$. D. degree in Information and Communication Engineering from SungKyun-Kwan University, Rep. of Korea in 2006. He is currently an assistant professor in Information and Communication Engineering, SKKU Institute for Convergence, and Center for Innovative Higher Education as Senior Researcher. His research interests are Big Data Platform, Intelligent Vehicular Network, Software Architecture and Design, and Datamining. 